\title{
Opportunities for Augmenting Conversation Through Technology for Persons with Dementia
}

\author{
Lorisa Dubuc and Alan Blackwell \\ Computer Laboratory, William Gates Building \\ University of Cambridge, $15 \mathrm{JJ}$ Thomson Avenue \\ Cambridge, CB3 OFD, UK \\ Lorisa.Dubuc@cl.cam.ac.uk; Alan.Blackwell@cl.cam.ac.uk
}

\begin{abstract}
In dementia care, difficulties in conversation are linked to a number of obstacles, for which we have developed a taxonomy comprised of four categories - cognitive, physical, social and identity. This paper describes current research being undertaken to ameliorate some of these obstacles using technology, including Project CIRCA and the Nostalgia project, as well as work currently being undertaken in Cambridge.
\end{abstract}

Augmentative and Alternative Communication (AAC), conversation, dementia, technology, cognitive psychology

\section{INTRODUCTION}

Dementia is a condition that is becoming increasingly prevalent as our society ages; according to the Alzheimer's Society [1], over 750,000 people in the UK are currently affected by dementia, with numbers on the rise. Looking worldwide, there are currently almost 18 million people living with dementia, and it is predicted that by 2025 there will be about 34 million people in this situation. Dementia is probably known best for the dramatic memory loss that accompanies it, but the difficulties associated with dementia are much more far-reaching and complex than many people realize.

In dementia care, there is often a focus on dealing with physical needs, as they are generally obvious and immediate. However, it is important to remember that individuals with dementia are still whole people, and have mental and emotional needs as well [2]. Partaking in communication, particularly conversation, is a valuable way to help an individual with dementia maintain a sense of control over their life, feel competent, express themselves, and keep their place in the world around them [3]. Furthermore, from the perspective of a caregiver, being able to continue having meaningful conversations with a friend or relative who has dementia will bring individuals closer together and ease some of the pain associated with cognitive decline for both parties as dementia progresses [4]. As normal conversation can be a difficult thing to achieve in dementia, there is an opportunity for technology to assist individuals in maintaining more satisfying relationships through the augmentation of conversation.

Our research focuses on the area of conversation, because although it is a common communication problem in dementia, it is one that has been given little attention. We will outline what a conversation entails for our purposes, how conversation can be valuable for those with dementia, why conversation problems happen in dementia, and what role technology can play in ameliorating these problems.

\section{CONVERSATION IS NOT JUST TALKING...}

\subsection{Conversation as a meaningful exchange}

While conversation is generally understood to be a verbal exchange of information between two or more people, Killick [5] points out that in dementia, "silence can be just as vital as communication". If non-verbal communication is primarily being used, such as eye contact, touching, or gesturing, it is possible that language may not be necessary to understand the meaning of the interaction; Killick [6] goes as far as to suggest that language might actually be an obstacle to communication in this type of interaction. Jane Crisp [7], in her interactions with her mother who had Alzheimer's, discovered the same thing; she suggests that the most important thing is remaining in touch, giving attention to one another, regardless of the degree of complexity involved in the interaction. Thus, we propose that for the duration of this paper, the term conversation will be used to denote meaningful contact between two or more individuals in which a connection is made through social interaction, whether verbal or nonverbal. 


\subsection{The Value of Conversation for People with Dementia}

While a diagnosis of dementia does not make a person any less of a person, associated difficulties do limit the activities an individual can partake in. Thus, it is important to build on the things that a person can still do, such as participation in social interactions and communication with other people. Clark and Witte [3] cite communication to be important in dementia for a variety of reasons, including as a "vehicle for expressing emotion, sharing memories, interacting socially, developing a supportive emotional atmosphere, stimulating awareness and responsiveness to the physical and human environment, and maintaining body awareness for activities of daily living". They go on to say that by encouraging communication in dementia, we are allowing the individual to maintain a purpose for living, as well as enjoy life through social interaction. Self-esteem and dignity are restored to the individual through successful communication, mitigating some of the losses they have experienced throughout the progression of their condition. Other reasons stressed by Alm et al. [2] for promoting communication in people with dementia are to try and avoid the dehumanizing way people who cannot communicate are treated, to prevent incorrect assumptions of the meaning of a person's behaviour by a caregiver (who may believe a person is being difficult when in fact something is wrong), and to retain skills that are still intact, due to the uneven decline of different skills in dementia.

Although there is still much to be learned about dementia and the experience of an individual affected by it, there have been many studies that have proven social interaction to be beneficial for a person's sense of self. According to Hagstrom [8], language (and therefore conversation) in dementia is used as a means to an end, as opposed to being an end in itself. While it might be said that the primary goal of using language is generally to convey information and have it understood, in dementia information is conveyed through language as a way to conduct a social interaction. It is the act of conversing which is valued, rather than the message sent, allowing the individual to have their personal identity validated. With a limited memory of recent events, it is very difficult for a person with dementia to update their sense of self. Conversation is one way to do this, to put part of themselves out into the world through interactions with others.

Finally, Savundranayagam et al. [9] mention that communication problems in dementia have been shown to activate problem behaviours, and cause undue stress on caregivers; thus, it is in a caregiver's best interest to make an effort towards conversation, with a possibility of seeing a reduction in behavioural problems and aggressiveness.

\section{OBSTACLES TO CONVERSATION WHEN DEALING WITH DEMENTIA}

There are many problems that accompany dementia, both in the physical and social experience of the individual. However, problems are not just limited to the person with dementia, as it takes two to have a conversation; family, friends and carers are also subject to a host of issues which complicate the pursuit of successful conversation. The combination of problems on both sides, as acknowledged by Goldsmith [10], creates numerous opportunities for misunderstanding and poor communication to occur. Before the creation of effective aids - technological or otherwise - can be considered, it is necessary to acknowledge the different causes of conversation breakdown for both parties. In particular, fragmented and difficult conversation is not always a sequela of the condition itself, but of factors associated with the condition through social and age correlations. Prior research has not identified a clear way to assemble these causes into logical groupings. We are advancing the field by constructing a taxonomy comprised of four categories - cognitive, physical, social, and identity - which can be used to understand the obstacles that hinder conversation in dementia (see Fig. 1).

\subsection{Cognitive Obstacles}

There are many cognitive problems that an individual with dementia must face over time as their dementia progresses. Dementia affects individuals in a variety of ways, such that a combination of difficulties could be experienced at any given time, or conversely, none at all. Most dominant is the issue of memory loss itself; day to day activities become increasingly difficult, recent events may be quickly forgotten, and eventually longer term memory begins to deteriorate as well. At different points, individuals may not recall what they did earlier in the day, what they are supposed to do next, or even who the person is with whom they are currently conversing [11].

In addition to general memory deficiencies, loss of language often occurs as time goes on. The individual may make functional errors (ie. difficulty with words and their meanings, use of excessive pauses when articulating), and pragmatic errors (ie. speaking too loudly, repeating questions, talking at inappropriate times), which can make conversation very slow and difficult for both parties [9]. Pauses may also encourage the other person to jump in with what they believe the person with dementia is trying to say, which may or may not be correct, but either way further reduces the individual's sense of independence and usefulness.

Other cognitive problems involved in conversation are difficulties in reasoning, getting distracted by sounds or objects nearby, and confabulation, which is "memory errors in which the person fabricates material instead of recalling information, but without conscious awareness of the fact" [11]. 


\subsection{Physical Obstacles}

Occasionally there are physical problems present unrelated to dementia which can hinder conversation; these must be addressed first in order to give conversation any chance of succeeding. If glasses are dirty or hearing reduced, individual may not see or hear the person talking to them. If further ailments are present, an individual may not be able to attend to the conversation due to resident pain [7]. If a caregiver is fatigued, the person with dementia will sense it and may think they have done something to elicit frustration, thus inhibiting conversation further. Reducing the observable interferences in conversation will increase the chance of messages being received in the first place, and make them available for processing and responding.

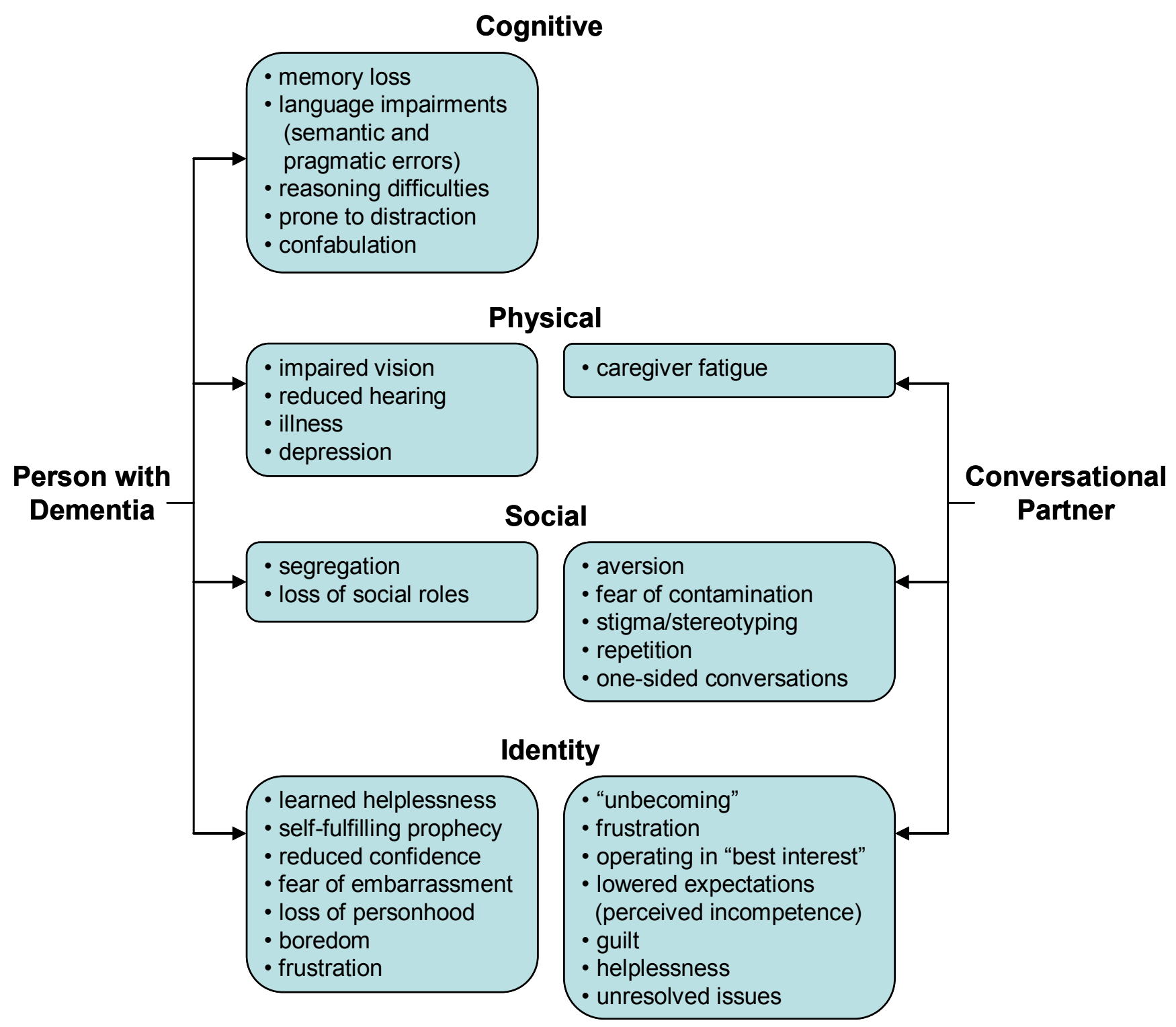

FIGURE 1: Taxonomy for classifying obstacles to conversation in dementia.

\subsection{Social Obstacles}

Apart from the physical symptoms of dementia, it has been suggested that the general perception and stereotype of the condition by society may lead to a reduction in conversational abilities. On the part of the conversational partner, there may be an aversion to other problems associated with dementia. There may also be fear of contamination from being around someone who is ill, or stigma and stereotyping of older persons. Presence of cognitive impairment in association with illness and old age often results in a double stigma associated with progressive dementias. However, if a person-centred approach is taken as suggested by Killick [6], these attitudes should be replaced by one acknowledging that a person with dementia is still a person, but with a debilitating illness; this would remove some of the fear of the condition and replace it with some hope for positive interaction. It has been argued by Goldsmith [10] that communication with people with dementia is indeed possible, but that an individual must believe this to be the case before it can be effectively attempted. 
Other problems encountered by people trying to carry on a conversation with individuals with dementia are the difficulties inherent in the conversation process itself. Dealing with repetitious questions and comments can become tiresome, particularly if a conversational partner wishes to treat conversation solely as a way to exchange information. According to Crisp [7], if they are able to look past this, they might find that repeated questions are actually an appeal for attention or reassurance, and may be able to respond in an appropriate manner. Conversations also tend to be very one-sided, as it may be difficult for the individual with dementia to remember what the other person just said, or they may not be able to attend to the incoming message as their resources are busy trying to formulate their next thought. This results in the other party becoming disinterested after a short period, and unlikely to want to continue the conversation for very long [8].

For the person with dementia, social isolation and segregation are common, as they become dependent on their carers and can no longer perform in the same activities that they did previously [2]. Additionally, they often experience a reduction in social roles, as they may no longer be able to fulfill some of the roles that they once did (ie. employee, volunteer, gardener, golfer). They become cut off from many of the social interactions that unimpaired individuals encounter on a daily basis, which limits their opportunities to exercise conversational skills and maintain those skills.

\subsection{Identity Obstacles}

Obstacles relating to identity and sense of self are varied in their causes, but all result in a reduced drive to communicate. Individuals with dementia may lose confidence in their voice over time, and develop a form of learned helplessness, as suggested by Lubinski [12]. She asserts that as a person with dementia interprets responses from others showing the inadequacy of their performance in conversation, they will begin to see their attempts as futile and will stop responding. This can happen through failed attempts using language, having sentences finished for them when they are not quick enough, or even by non-verbal messages from their conversational partner expressing discontent and frustration. Clark and Witte [3] remark that a person with dementia, when confronted with feelings of inadequacy regarding their ability to communicate, will often withdraw from interactions. This ties in to the concept of self-fulfilling prophecy, as described by Adler and Rodman [13], wherein the less they respond makes it even less likely that others will attempt to engage them in conversation, thinking them incompetent; this results in a vicious cycle that is hard to break out of. Furthermore, other emotions such as pride, embarrassment, boredom or frustration over declining abilities may also discourage a person from making an attempt at conversation.

As the condition progresses, it may seem as though there is less and less of the original person still present; in many situations, this "dehumanizing" or "un-becoming" effect causes people close to the person with dementia to shy away from interactions with them, believing that they are becoming less of a person [10,14,15]. Even if this isn't the case and a caregiver maintains a person-centred outlook regarding dementia, it is still possible for them to have lowered expectations of what the person with dementia is capable of, and treat them like a child [15]. Strauss [16] also mentions that deep-rooted emotions may keep family or friends from initiating conversation, such as frustration, guilt and helplessness over an inability to help the person with dementia get better, and feelings about unresolved issues which were present before dementia began (and now may never get a chance to be resolved).

Despite the obvious problems which can inhibit conversation when dealing with individuals with dementia, we need to keep aware of all the benefits that efforts to stimulate conversation bring. As Goldsmith [10] points out, while there are inevitable problems that do arise as a result of the condition, they do not mean that communication cannot successfully happen; we need to recognize what a person with dementia can still do, and build upon that. Thus, ways need to be found to circumvent the negative issues and inspire conversation, on both the part of the person with dementia and the people around them.

\section{THE ROLE TECHNOLOGY CAN PLAY IN ENCOURAGING CONVERSATION IN DEMENTIA}

It is clear from this analysis that social interaction and communication is of great importance in maintaining an individual's sense of self and place in the world. It is therefore important that work be done to try and improve the possibility of meaningful interactions taking place. This can be achieved by working towards stimulating and improving the process of conversation between individuals with dementia and the people around them, whether they are carers, family, friends, or other people with the same condition. There are a variety of approaches used in an attempt to ameliorate the problems described, many of which are performed through social, educational, or other means; however, our main focus here is technological intervention. While there are many individual and unique ways that people currently attempt to manually do this, we believe that it is an important priority to extract this knowledge and apply it in such a way that many people are able to benefit from it. There are a number of projects currently pursuing the goal of improving conversation through technology, as technology offers consistent storage and retrieval of such information along with diverse interaction methods. 


\subsubsection{Project CIRCA (Computer Interactive Reminiscence and Conversation Aid)}

A collaboration involving researchers from both Dundee University and St. Andrews University, Project CIRCA is well-known for its novel approach using technology-led reminiscence to encourage conversation between people with dementia and their caregivers. Gowans et al. [14] describe CIRCA as using computer-based interactive multimedia in conjunction with a touch-screen to encourage both verbal and non-verbal communication through the stimulation of long-term memory. Acting as an electronic scrapbook, various types of media were presented in three subject areas, and individuals were encouraged to explore the pages and talk about the topics as they pleased.

Applying our taxonomy, this project most strongly compensated for the cognitive obstacles in dementia by not requiring the user to remember what they were previously speaking about or what they had wanted to talk about next; the aim was to explore and enjoy the material. There was support for language difficulties where reading was concerned, as some information was also relayed through audio, but it is possible that problems with comprehension or expressing oneself through language could still inhibit conversation. The physical obstacles were considered, although occurring later in the project as a result of user trials; in order to make it more comfortable for an older person to use, the researchers decided to shift the controls to the bottom of the screen, and to mount the display on an adjustable stand. Social obstacles were not removed for the person with dementia, as the system did not provide an opportunity for individuals to integrate more with the general populous, nor did it increase their chances of developing new or improved social roles. Similarly, problems which inhibit conversation from the caregiver's point of view still remained, as nothing was done to help integrate them into the conversation or remove the stigma associated with dementia. It is possible though that through witnessing the spontaneous conversation occur, some of the aversion and stigma may have dissipated. Finally, identity obstacles were strongly addressed by presenting the individuals with information they were familiar with via reminiscence; this allowed caregivers the chance to experience a spontaneous, unprepared conversation with the individual, to potentially see a new glimpse of the person through untold stories, as well as allowing the individual with dementia to take control of the conversation with confidence [2].

\subsubsection{The Nostalgia Project}

Another project which has encouraged conversation through technology is entitled Nostalgia; an old-style radio combined with an interactive tabletop interface were used to allow older individuals to listen to old music and news from the twentieth century. Although the users of this prototype were elderly users without dementia, the purposes of the project are parallel to the ones we have already discussed: increasing social interaction, successfully integrating a form of usable technology into the lives of older adults, and providing reminiscent information. The researchers found that the patients were curious about the technology and were interested in interacting with it; conversation quickly ensued between the patients about what they were hearing, as well as singing and some emotional displays [17].

Comparing the obstacles addressed with those seen in CIRCA, the conversational obstacles mediated by this project are very similar in both strengths and weaknesses. Physical obstacles weren't explicitly mentioned, although user studies were conducted prior to finalizing the design, in order to make the design aesthetically pleasing and easy to interact with. Social obstacles were not addressed, as the elderly individuals were interacting with the system in the communal area of a nursing home; within this group, many of the social obstacles that would normally be seen may not exist, as a level of understanding of others' conditions would be inherent to those present. Lastly, a reduction in the obstacles relating to identity correlate with those seen in CIRCA, as individuals were able to hear fresh narratives from those around them, as well as build confidence through expressing themselves to others.

\subsubsection{Other Related Work}

There has also been some work done in the area of conversation generation and augmentation for aphasiacs as opposed to persons with dementia. For example, technology has been leveraged in the creation of a communication service to help non-speaking people socially interact using pictures and other media to share stories [18]. Additionally, a word-based system called TalksBac has been developed to store personal sentences and stories for a user, and offer probable selections later for retrieval during conversation [19]. These two projects, in addition to other work going on in this area, are also attempting to turn conversation into a more enjoyable, less stressful prospect for those involved.

\subsection{Current Work in Cambridge}

There are several projects currently underway in Cambridge, employing a variety of research methods. We have chosen to direct our research towards interactions with users, in order to benefit from a participatory design perspective. This has allowed us the opportunity to understand first-hand the conversational difficulties encountered in dementia, and to refine the taxonomy in Fig.1. 


\subsubsection{Participation in Local Art Group}

One of us (Dubuc) has joined with the local Alzheimer's Society branch in Cambridge, where she has been involved in many facets of their programme. One such activity is a weekly art group that she has been attending, comprised of both persons with dementia and their carers. The art group is a unique and accommodating environment for those with language difficulties, as it allows a way for communication to still flourish through nonverbal modes such as drawing and painting. There is no pressure to talk, as art becomes the main focus, but conversation generally ensues based on the shared task. Attending a group such as this eases social obstacles often experienced with dementia through integration and inclusion, improves personhood through increased confidence, and updates personal identity through learning and using new creative skills. This has given us the opportunity to observe how individuals interact with one another, to share in conversations with people with dementia, and see how particular communication difficulties are handled as they arise.

\subsubsection{Roadshows}

Another unique activity designed to spend time with individuals with dementia is in the form of roadshows, which entails visiting market towns around Cambridgeshire to distribute information about Alzheimer's and talk with people affected by the condition in various areas. Taking place this summer, the roadshows will allow us the opportunity to discuss communication and technology with people across the region, to understand which of the obstacles to conversation as outlined above are most common, and to recruit individuals to take part in further research.

\subsubsection{Focus Groups with Caregivers}

A further pursuit soon to be implemented is the creation of a focus group comprised of carers, carefully selected by the Alzheimer's Society, who have dealt with communication issues during their experience with dementia. This group will serve two purposes, with a primary focus being to provide a therapeutic environment for caregivers to discuss any problems they are having. By observing and listening to problems as they are articulated, we will gain an understanding of common communication issues that arise, and some of the makeshift solutions used to deal with them. The second purpose will be to discuss possible applications of technology for progressive memory loss, getting away from the stereotypical idea of technology being a computer, mouse and keyboard. Activities will include demonstrating possible technologies to assist someone with dementia with conversation, and discussing ideas for how caregivers might see technology being applied based on their experiences. Furthermore, as the focus group will be a monthly gathering, any ideas generated by this group can be taken away for further thought and development, and brought back at a later date for validation and refinement.

\subsubsection{Other work in Cambridge}

While our work thus far has focused primarily on interactions with users, the Interactive Systems Group at Microsoft Research Cambridge has begun clinical trials with memory impaired users using a small, portable camera they call "SenseCam". SenseCam is attached to the body using a neck strap, and is comprised of a small camera with a number of sensors [20]. The focus is on using the technology to help people with both acquired and degenerative memory impairments consolidate recent autobiographical memory through rehearsal. As well, the information collected produces a pictorial diary of autobiographical experiences which can be accessed any time, to be used for reassurance, reminders, or as a fun activity unto itself [21]. In addition to helping individuals remember daily events, a potential outcome of this project will be improved personhood through an updated sense of self, enhanced social interactions, and increased confidence. There is regular contact between these two projects, as the goals are somewhat similar in nature.

\section{CONCLUSIONS}

While there has been some work done in augmenting conversation through reminiscence for persons with dementia and supplemental speech technology created for aphasiacs, there has been relatively little work directly addressing the possibilities technology can offer in relation to specific conversational problems associated with progressive memory loss. We have recognized this need, thus the next phase of our work will be focusing on the design of technology in this area, taking into account and addressing the conversational obstacles presented in the different areas - cognitive, physical, social, and identity. As we are fully aware of the difficulties involved due to the variety and variability of problems present in any particular case of dementia, we recognize that generalizations need to be drawn and applied in order to create technological aids able to successfully augment conversation. We are undertaking work in this area as we think it's important for not only the social value that it may bring, but also for the impact the results may have beyond this specific area. For example, our work is sponsored in part by Boeing, who are interested in the broader impact that communication-support technologies might have for other populations. As interest and work in this area increase in the future, so will the potential for easier and more meaningful interactions for both the person with dementia and their conversational partners. 


\section{ACKNOWLEDGEMENTS}

We would like to acknowledge support from Boeing, the Cambridge Trusts, Universities UK, Barry Plumpton and the Cambridge branch of the Alzheimer's Society, and Linda Clare.

\section{REFERENCES}

[1] Alzheimer's Society UK. (2004) Policy Positions: Demography. Retrieved April 9, 2005, from http://www.alzheimers.org.uk/News_and_Campaigns/Policy_Watch/demography.htm

[2] Alm, N., Astell, A., Ellis, M., Dye, R., Gowans, G., and Campbell, J. (2004) A cognitive prosthesis and communication support for people with dementia. Journal of Neuropsychological Rehabilitation, 14(1/2), 117-134.

[3] Clark, L. and Witte, K. (1995) Nature and Efficacy of Communication Management in Alzheimer's Disease. In R. Lubinski (ed.), Dementia and Communication, pp. 238-254. Delmar, London.

[4] Morris, M., Lundell, J., Dishman, E., and Needham, B. (2003) New perspectives on ubiquitous computing from ethnographic study of elders with cognitive decline. In Proceedings of UbiComp 2003: Ubiquitous Computing, $5^{\text {th }}$ International Conference, pp. 227-242. Springer, Seattle.

[5] Killick, J. (1999) 'Dark Head Amongst the Grey': Experiencing the Worlds of Younger Persons. In S. Cox and J. Keady (Eds.), Younger People with Dementia: Planning, Practice and Development, pp. 153-172. Jessica Kingsley Publishers, London.

[6] Killick, J. and Allan, K. (2001) Communication and the care of people with dementia. Open University Press, Buckingham.

[7] Crisp, J. (2000) Keeping in touch with someone who has Alzheimer's. Ausmed Publications, Melbourne.

[8] Hagstrom, F. (1995) Conversational Repetitions and Dementia: An Activity Theory Analysis. In K. Junefelt (ed.), Proceedings of the XIVth Scandinavian Conference of Linguistics and the VIIIth Conference of Nordic and General Linguistics, August 16-21 1993. Gothenburg Papers in Theoretic Linguistics 73, pp. 41-52. Gothenburg University Press, Göteborg.

[9] Savundranayagam, M. Y., Hummert, M. L., and Montgomery, R. J. V. (2005) Investigating the Effects of Communication Problems on Caregiver Burden. Journal of Gerontology: Social Sciences, 60B, S48-S55.

[10] Goldsmith, M. (1996) Hearing the Voice of People with Dementia: Opportunities and Obstacles. Jessica Kingsley Publishers, London.

[11] Miller, E., and Morris, R. (1993) The Psychology of Dementia. John Wiley \& Sons Ltd, Chichester.

[12] Lubinski, R. (1995) Learned Helplessness: Application to Communication of the Elderly. In R. Lubinski (Ed.), Dementia and Communication (pp. 142-151). Delmar, London.

[13] Adler, R. B. and Rodman, G. (2003) Understanding Human Communication (8th ed.). Oxford University Press, Oxford.

[14] Gowans, G., Campbell, J., Alm, N. Astell, A., Ellis, M., and Dye, R. (2004) Designing a Multimedia Conversation Aid for Reminiscence Therapy in Dementia Care Environments. In $\mathrm{CHI}$ '04: $\mathrm{CHI}$ '04 extended abstracts on Human factors in computing systems, pp. 825-836. ACM Press, Vienna.

[15] Kitwood, T. (1997) Dementia Reconsidered: The person comes first. Open University Press, Buckingham.

[16] Strauss, C. (2001) Talking to Alzheimer's: Simple Ways to Connect When You Visit with a Family Member or Friend. New Harbinger Publications, Oakland, CA. 
[17] Nilsson, M., Johansson, S., and Hắkansson, M. (2003) Nostalgia: An Evocative Tangible Interface for Elderly Users. In CHI '03: $\mathrm{CHI}$ '03 extended abstracts on Human factors in computing systems, pp. 964-965. ACM Press, Ft. Lauderdale.

[18] Hine, N. and Arnott, J. L. (2002) Assistive social interaction for non-speaking people living in the community. In V. L. Hanson and J. A. Jacko (Eds.), Proceedings of the ACM Conference on Assistive Technologies, ASSETS 2002, pp 162-169. ACM Press, Edinburgh.

[19] Waller, A., Dennis, F., Brodie, J., and Cairns, A.Y. (1998) Evaluating the use of TalksBac, a predictive communication device for nonfluent adults with aphasia. International Journal of Language \& Communication Disorders, 33(1), 45-70.

[20] Gemmell, J., Williams, L., Wood, K., Lueder, R., and Bell, G. (2004) Passive Capture and Ensuing Issues for a Personal Lifetime Store. In CARPE'04: Proceedings of the the 1st ACM workshop on Continuous archival and retrieval of personal experiences, pp. 48-55. ACM Press, New York.

[21] Quested, T. (15 June 2004) Microsoft research foray lauded by Alzheimer's Society director. Business Weekly UK. Retrieved April 8, 2005, from http://research.microsoft.com/hwsystems/sensecam business weekly.mht 